\title{
As faces da violência obstétrica no âmbito hospitalar
}

\author{
The faces of obstetric violence in the hospital environment \\ Los rostros de la violencia obstétrica en el ámbito hospitalario
}

Adilson Mendes de Figueiredo Júnior ${ }^{1 *}$, Alda do Socorro Cardoso Moraes ${ }^{1}$, Clebson Luis Trindade da Silva ${ }^{2}$, Juliana de Souza Cobel $^{2}$, Kamyla Pinto Pinho², Maria de Jesus Evangelista Pimentel ${ }^{2}$, Carlos Kayque Araújo da Silva ${ }^{3}$, João Victor Tavares da Costa ${ }^{3}$, Paula Amanajás de Melo ${ }^{4}$, Sabrina Macambira Guerra da Rocha 5 .

\section{RESUMO}

Objetivo: Realizar um levantamento sobre os tipos de violência obstétrica que ocorrem em mulheres durante a assistência ao parto no âmbito hospitalar. Métodos: $O$ estudo foi do tipo qualitativo descritivo com o intuito de realizar um levantamento dos principais tipos de violência obstétrica que ocorrem durante a assistência ao parto em âmbito hospitalar através de uma Revisão Integrativa da Literatura. Resultados: Verificaram-se as condutas adotadas após a ocorrência da violência obstétrica durante a assistência ao parto e quais as intervenções que podem ou são realizadas pelo enfermeiro de acordo com a literatura. Considerações finais: É necessário o treinamento de profissionais da saúde para promover um atendimento humanizado e adequado para cuidados de saúde da mulher. Portanto, há necessidade de estudos que destacar a questão entre os profissionais de saúde, com ênfase nas boas práticas incluídas nele e conformidade e conformidade com leis, punições e formas de denúncia, para promover a reflexão sobre eles e adoção de comportamentos mais profissionais e aceitáveis.

Palavras-chave: Parto humanizado, Saúde, Enfermagem.

\begin{abstract}
Objective: Conduct a survey on the types of obstetric violence that occur in women during childbirth care in the hospital. Methods: The study was of a descriptive qualitative type in order to carry out a survey of the main types of obstetric violence that occur during childbirth care in the hospital through an Integrative Literature Review. Results: The behaviors adopted after the occurrence of obstetric violence during childbirth care and which interventions that can or are performed by nurses were verified according to the literature. Final considerations: Training of health professionals is necessary to promote humanized and adequate care for women's health care. Therefore, there is a need for studies that highlight the issue among health professionals, with an emphasis on good practices included in it and compliance and compliance with laws, punishments and forms of reporting, to promote reflection on them and adoption of more professional and acceptable behaviors.
\end{abstract}

Key words: Humanized childbirth, Health, Nursing.

\footnotetext{
1 Universidade do Estado do Pará (UEPA), Belém - PA. *E-mail: adilsonmdfj@hotmail.com

2 Escola Superior da Amazônia (ESAMAZ), Belém - PA.

${ }^{3}$ Centro Universitário da Amazônia (UNIFAMAZ), Belém - PA.

${ }^{4}$ Universidade da Amazônia (UNAMA), Belém - PA.

5 Universidade Federal do Pará (UFPA), Belém - PA.
}

SUBMETIDO EM: 3/2021

ACEITO EM: 3/2021

PUBLICADO EM: 4/2021 


\section{RESUMEN}

Objetivo: Realizar una encuesta sobre los tipos de violencia obstétrica que se presentan en las mujeres durante la atención del parto en el hospital. Métodos: El estudio fue de tipo descriptivo cualitativo con el fin de realizar un relevamiento de los principales tipos de violencia obstétrica que ocurren durante la atención del parto en el hospital a través de una Revisión Integrativa de la Literatura. Resultados: Se verificaron las conductas adoptadas luego de la ocurrencia de violencia obstétrica durante el cuidado del parto y qué intervenciones que pueden o son realizadas por enfermeras de acuerdo a la literatura. Consideraciones finales: La formación de los profesionales de la salud es necesaria para promover una atención humanizada y adecuada a la salud de la mujer. Por ello, se necesitan estudios que resalten el tema entre los profesionales de la salud, con énfasis en las buenas prácticas incluidas en el mismo y el cumplimiento y cumplimiento de las leyes, sanciones y formas de denuncia, para promover la reflexión sobre las mismas y la adopción de más profesionales y comportamientos aceptables.

Palabras clave: Parto humanizado, Salud, Enfermería.

\section{INTRODUÇÃO}

A violência obstétrica é genericamente tratada como violência contra a mulher. Porém esta violência em específico é cometida em um dos momentos mais importantes da vida de uma mulher, no nascimento do seu filho, um momento sonhado, idealizado e por muitas vezes conquistado. E, é deste momento em que a parturiente é incapaz de se defender ou fazer com que sua vontade e/ou dignidade sejam respeitadas, que alguns profissionais da saúde se aproveitam e fazem comentários agressivos, ofensas, ameaças, discriminação racial e/ou socioeconômica, exames de toque abusivos, agressão física, tortura física e/ou psicológica, entre outros (PAULETTI M, et al., 2020).

Estas violências são silenciadas, pois ainda existe nas mulheres a cultura de se sentirem culpadas, e muito mais que isso, a parturiente que sofre violência obstétrica se apega a felicidade do nascimento do seu bebê e acaba por deixar para lá a violência por ela sofrida na intenção de esquecer, não levando adiante uma denúncia. Hoje, porém com a evolução das mídias sociais que propagam muita informação, as mulheres conseguem ver que não estão sozinhas, e que por maior que seja a felicidade de ter seu bebê com vida ali nos seus braços, a violência por elas sofrida nunca se apagará de suas memórias e ficará marcada como uma cicatriz na sua alma (CAMPOS VS, et al., 2020).

Muitos estudos revelaram e reconhecem o tratamento desrespeitoso, ofensivo e até negligente para com mulheres durante o parto em unidades de saúde em várias partes do mundo. Vários desses estudos referemse à violência física, violência psicológica, abuso verbal, procedimentos realizados sem consentimento ou com informações limitadas, falta de privacidade e outros procedimentos de cuidados maternos não recomendados (CURI PL, et al., 2020).

Apesar destes tipos de evidência, um consenso sobre o caminho a seguir não foi alcançado a nível internacional. Em 1985, a Organização Mundial da Saúde publicou uma série de recomendações sobre assistência ao parto, boas práticas e desencorajando aqueles que não tiveram nenhum benefício ou foram prejudiciais ao paciente, afirmando o seguinte (MENEZES FR, et al., 2020).

Por esse motivo, a prevalência de violência obstétrica é desconhecida; portanto, seu impacto na saúde e bem-estar desta população vulnerável grupo de mulheres não pode ser determinado. Uma investigação profunda é necessário e espera-se que contribua para uma visualização aprimorada o problema. Além disso, tal investigação é essencial para quantificar a violência obstétrica com o objetivo de buscar estratégias que permitir sua prevenção e possível erradicação (SILVA TM, 2020).

Baseando-se na importância do resgate do papel principal da mulher durante o parto e na crescente especulação de hospitais ditos como "humanizados," além do respeito às recomendações da OMS e Ministério da Saúde do Brasil, considera-se importante levantar as questões inerentes a violência obstétrica nas instituições hospitalares públicas brasileiras (ALTHABE F, et al., 2014). 
Independente de políticas ou tendências, a mulher é a figura principal no momento do parto, sendo ela inserida como centro das atenções, sujeito do processo da parturição. Muitas práticas foram estabelecidas sem apoio científico na obstetrícia e são utilizadas até os dias atuais. Porém, estudos demonstraram que tais práticas são prejudiciais e que devem ser utilizadas com cuidado ou até mesmo banidas da assistência (ARAUJO MMT e SILVA MJP, 2017).

A definição de violência obstétrica relaciona-se a toda forma de ato desumano e/ou abuso de procedimentos desnecessários que interferem negativamente a vida das mulheres. Importante perceber que a violência obstétrica é considerada outra forma da violência de gênero contra a mulher (SOUZA ACAT, et al., 2019).

Ao longo dos anos, a violência obstétrica foi normalizada e aceita por algumas populações. No entanto, muitos esforços estão sendo feitos em todo o mundo para reconsiderar o parto como um processo fisiológico, os principais protagonistas são a mãe e o neonato, e onde o pessoal de saúde deve estar atento aos riscos, minimizando intervenções desnecessárias. Também é importante enfatizar que a violência obstétrica é exercida não apenas sobre as mulheres e seus neonatos, mas também sobre seus companheiros e familiares (MARQUES GM e NASCIMENTO DZ, 2019).

Pesquisar sobre a violência obstétrica, vem trazer para a sociedade um assunto muito importante a ser debatido, uma vez que suas consequências trazem malefícios físicos e psicológicos nas mulheres que o sofrem, refletindo de modo único na vida de uma família, causando doenças físicas e psicológicas que marcaram sua vida para sempre. É importante aos acadêmicos enquanto pesquisadores, observar e buscar as necessidades e problemas que a sociedade enfrenta antes dela própria, sempre com o objetivo da busca de soluções (NASCIMENTO SL, et al., 2019).

O direito através da Constituição da República Federativa do Brasil de 1988 prevê o respeito à dignidade da pessoa humana e o direito à vida entre seus fundamentos, na condição de garantidor de direitos, este profissional irá facilitar o entendimento do problema de forma objetiva. Com o reconhecimento do Estado, através de legislação específica sobre o assunto a sociedade profissional de modo geral se posicionará de forma mais humana após o reconhecimento do fato social em questão (ALTHABE F, et al., 2014).

De acordo com Nascimento SL, et al. (2019), a efetuação de procedimentos desnecessários é também um exemplo de violação do direito humano de estar livre de tratamentos cruéis, humilhantes e degradantes. A violência obstétrica seja ela física, psicológica, verbal é muitas vezes realizada com consentimento da mulher por desconhecer alguns procedimentos. Esse fato também deve ser levado em consideração, pois os procedimentos podem acarretar alterações além de outras complicações para o resto da vida (OLIVEIRA LLF, et al., 2019).

O objetivo do atual estudo foi realizar um levantamento sobre os tipos de violência obstétrica que ocorrem em mulheres durante a assistência ao parto no âmbito hospitalar.

\section{MÉTODOS}

O estudo foi do tipo qualitativo descritivo com o intuito de realizar um levantamento dos principais tipos de violência obstétrica que ocorrem durante a assistência ao parto em âmbito hospitalar. Como critério de inclusão foram usadas publicações de 2015 a 2020 (intervalo de 5 anos) em acervos nacionais e internacionais de pesquisa, Scientific Electronic Library Online (SCIELO), Google acadêmico, Literatura Latino-americana e do Caribe em Ciências da Saúde (LILACS), entre outros, que tratam sobre a temática e deram suporte para responder aos objetivos do estudo. Como critérios de exclusão, foram excluídos trabalhos internacionais que não falavam sobre a temática, não se enquadraram no recorte temporal de dez anos de pesquisa e não foram gratuitos e encontrados nas bases das pesquisas.

Os dados depois de obtidos foram analisados através da descrição das pesquisas e da análise da revisão de literatura. Na literatura descritiva temos a coleta, organização e descrição dos dados, que foi realizada por meio de quadros e de análises descritivas. 


\section{RESULTADOS}

Realizou-se um levantamento sobre os tipos de violência obstétrica que ocorrem em mulheres durante a assistência ao parto em âmbito hospitalar. Para isso, traçou-se o perfil dos artigos (Quadro 1).

Quadro 1 - Trabalhos bases à análise e discussão dos resultados.

\begin{tabular}{|c|c|c|}
\hline Título & Objetivo & Ano \\
\hline $\begin{array}{l}\text { Violência obstétrica: a dor que } \\
\text { cala. }\end{array}$ & $\begin{array}{l}\text { Retratar a violência obstétrica sofrida por mulheres no pré- } \\
\text { parto e parto, a partir do relato de suas vivências. }\end{array}$ & 2018 \\
\hline $\begin{array}{l}\text { A comunicação com o paciente } \\
\text { em cuidados paliativos: } \\
\text { valorizando a alegria e o } \\
\text { otimismo. }\end{array}$ & $\begin{array}{l}\text { Conhecer as expectativas de pacientes em cuidados } \\
\text { paliativos em relação à comunicação com as pessoas da } \\
\text { equipe de enfermagem. }\end{array}$ & 2020 \\
\hline $\begin{array}{l}\text { Gênero no direito internacional: } \\
\text { Discriminação, violência e } \\
\text { proteção. }\end{array}$ & $\begin{array}{l}\text { Autorexplicar como poucos a violência de gênero, familiar e } \\
\text { doméstica, perpassando um dosprobemas mais graves na } \\
\text { investigação, aspecto conceitual. }\end{array}$ & 2020 \\
\hline $\begin{array}{l}\text { Conduta no trabalho de parto e } \\
\text { parto normal. }\end{array}$ & $\begin{array}{l}\text { Reunir informações resumidas sobre a elaboração, a } \\
\text { metodologia e as recomendações das Diretrizes Nacionais } \\
\text { de Assistência ao Parto, no sentido de dar transparência e } \\
\text { acesso rápido e fácil para toda a sociedade sobre o resultado } \\
\text { desse trabalho }\end{array}$ & 2016 \\
\hline $\begin{array}{l}\text { Saúde da mulher e enfermagem } \\
\text { obstétrica. }\end{array}$ & $\begin{array}{l}\text { Contextualizar a enfermagem obstétrica em relação às metas } \\
\text { do Milênio, traçadas pela Organização Mundial da Saúde em } \\
\text { parceria com as mais destacadas associações profissionais } \\
\text { vinculadas à saúde da mulher e do recém-nascido, em níveis } \\
\text { mundial e local. }\end{array}$ & 2019 \\
\hline $\begin{array}{l}\text { Humanização da atenção ao } \\
\text { parto e nascimento no Brasil: } \\
\text { pressuposto para uma nova ética } \\
\text { na gestão e no cuidado. }\end{array}$ & $\begin{array}{l}\text { Propor reflexões sobre o modelo de atenção e gestão ao } \\
\text { parto e nascimento no Sistema Único de Saúde, } \\
\text { considerando contribuições da Política Nacional de } \\
\text { Humanização (Humaniza SUS), na perspectiva da reversão } \\
\text { do atual panorama de assistência, caracterizado pela } \\
\text { submissão da mulher ao saber biomédico, aquilo que se tem } \\
\text { designado de parto tecnocrático, que pouco se vale de } \\
\text { evidencias cientificas }\end{array}$ & 2019 \\
\hline $\begin{array}{l}\text { Legislação profissional e marcos } \\
\text { regulatório da prática assistencial } \\
\text { da enfermeira obstétrica no } \\
\text { sistema único de saúde } \\
\text { obstetrícia. }\end{array}$ & $\begin{array}{l}\text { Ser um guia acerca das legislações profissionais e dos } \\
\text { dispositivos legais do Sistema Único de Saúde que regula } \\
\text { a atuação da enfermeira obstétrica nos diversos cenários de } \\
\text { atenção à saúde da mulher, elucidando acerca das suas } \\
\text { atribuições legalmente constituídas }\end{array}$ & 2020 \\
\hline $\begin{array}{l}\text { Violência Obstétrica: Mulheres } \\
\text { são Seres Humanos, não } \\
\text { Animais. }\end{array}$ & $\begin{array}{l}\text { Analisar as percepções das mulheres acerca da assistência } \\
\text { obstétrica no que se refere ao atendimento de seus direito } \\
\text { de acesso ao serviço de saúde durante o processo de parto } \\
\text { e nascimento }\end{array}$ & 2018 \\
\hline $\begin{array}{l}\text { Uma Dor Além Do Parto: } \\
\text { Violência Obstétrica em Foco. } \\
\text { Revista de direitos humanos e } \\
\text { efetividade. }\end{array}$ & $\begin{array}{l}\text { Discutir a violência obstétrica, bem como sua } \\
\text { caracterização, a partir da análise dos acórdãos do Supremo } \\
\text { Tribunal Federal (STF) e Superior Tribunal de Justiça (STJ). }\end{array}$ & 2016 \\
\hline
\end{tabular}

Fonte: De Figueiredo Júnior AM, et al., 2021. 


\section{DISCUSSÃO}

Fatores sociais como baixa escolaridade, dificuldades de acesso ao hospital, fatores obstétricos como ausência de assistência pré-natal e multiparidade, são estatisticamente significativos associados a um risco aumentado de parto domiciliar (OLIVEIRA MSS, et al., 2019). O baixo status socioeconômico foi encontrado na literatura como um fator que impede a assistência ao parto da maneira como deveria ser. Em um estudo brasileiro, $41 \%$ das mães que não deram à luz explicaram que não podiam pagar a conta do hospital e 31\% disseram que tinham possibilidades de transporte inadequadas (MARQUES SB, et al., 2020).

Em um estudo econômico das Filipinas, foi relatado que o coeficiente de elasticidade sugere que uma redução de $1 \%$ no tempo médio de viagem a estabelecimentos públicos modernos aumentará a probabilidade de escolher essa opção para parto em 1,2\%. Esses estudos estão de acordo com o nosso estudo. Mesmo assim, ao interpretar o significado e as implicações dessas descobertas, enfrentamos argumentos que aconselham cautela (TEIXEIRA PC, et al., 2020).

Segundo Oliveira MSS, et al. (2012) o contexto histórico e cultural da sociedade afeta de forma direta a mulher, trazendo resultados classificáveis como negativo, sem perder o objetivo, junto com os movimentos pelo parto humanizado, vencer essa árdua luta, é visível a real necessidade de um novo modelo relacionado ao atendimento obstétrico, baseado nos direitos humanos, vale ressaltar a importância de ter como pauta principal dos debates o empoderamento das mulheres sobre parto, sexualidade e o próprio corpo, sem deixar de falar da importância da implementação de leis, objetivando o combate a violência obstétrica.

A relevância de nossas descobertas para o contexto geral da política de assistência à saúde da maternidade não é direta, e a extensa revisão da literatura sobre o local de parto e seus determinantes teve o objetivo de mostrar a dicotomia entre hospital e parto domiciliar como uma questão importante de pesquisa. A literatura sobre indicadores de processo será de especial e alta importância nesse contexto e pode nos guiar quando procurarmos especificamente respostas para remover as barreiras à obtenção de saúde materna durante o parto. Isso poderia ser feito incorporando uma abordagem qualitativa em nossos esforços de pesquisa (CUNHA AL, et al., 2019).

Variáveis econômicas como status de pontuação de comodidade e distância da maternidade são os principais componentes causais para a escolha do local de parto, enquanto características culturais como etnia, observância dos dias rituais da menarca e tipo e tamanho da família, ou quem é o chefe da família ou a história obstétrica têm pouca influência ou não são indicadores de risco para o local de parto (CASTRO ATB e ROCHA SP, 2020).

Concluímos, portanto, que as mães e as famílias, para chegar a uma "tomada de decisão fundamentada", como postulado por Cunha AL (2019), pode estar constantemente pensando no ônus de sua decisão sobre a família de comparecer ao hospital para parto. Assim, o baixo status socioeconômico e a longa distância física de mais de uma hora da maternidade agem como barreiras ao parto.

A distância física da maternidade, juntamente com a educação da mãe e o status de pontuação de comodidade da família podem servir como indicadores do status socioeconômico em um país em desenvolvimento, pelo menos ao procurar status socioeconômico e assistência à maternidade (PROGIANTI JM, et al., 2019).

Em relação à violência obstétrica na assistência ao parto os estudos mostram como aspectos da desumanização: práticas discriminatórias (barreiras morais e religiosas), tratamento indigno (uso de linguagem severa e grosseira), negligência (demorando muito para sofrer evacuação uterina) e proibição de acompanhamento, entre outras atitudes institucionais dos profissionais de saúde (ALTHABE F, et al., 2014).

Os resultados encontrados relacionados à violência obstétrica caracterizada pela desumanização demonstram a congruência com a ideia desenvolvida que considera a violência como um ato desprovido de humanidade, tratando outra pessoa como objeto, descrevendo a necessidade de se humanizar como um devir eterno, que requer atualização a todo o momento de ação e em todo relacionamento estabelecido (TEIXEIRA PC, et al., 2020). 
Ainda nesse contexto, a prática da medicalização e patologização de processos naturais são entendidas como um fenômeno antigo em que a medicina controla a sociedade através de regras de conduta e padrões que influenciam comportamentos humanos. Esse fenômeno começa a reescrever eventos fisiológicos e comportamentos sociais, referindo-os a intervenções de práticas especializadas, interferindo e desnaturalizando a ação independente e racional dos seres em sua própria produção em saúde (PASCHE DF, et al.,2010).

Os artigos identificaram fatores associados ao aleitamento materno na primeira hora de vida mostraram que ter uma cesariana e não ficar em uma sala comum após o parto é fator que negativamente interferiu no vínculo estabelecido entre a mãe e o recém-nascido. É importante destacar que a taxa de $61,5 \%$ de cesáreas apresentada pelo estudo rompe com o preconizado pela Organização Mundial da Saúde em relação à adoção desta cirurgia (NASCIMENTO SL, et al., 2019).

No momento do parto, a principal necessidade das mulheres é o apoio emocional. O parto é um processo fisiológico que tem o início e a evolução por si só: o colo do útero começa a abrir, o útero é contraído, o bebê começa a descer e tudo fica mais intenso, até o momento do nascimento. Todo esse processo pode acontecer de maneira confortável e segura, sem intervenções. As contrações uterinas, a liberação de hormônios e a passagem pela vagina favorecem líquidos e uma melhor adaptação ao mundo (PAULETTI M, et al., 2020).

$O$ direito das mulheres à informação e escolha, o apoio emocional durante o parto, o primeiro contato da mãe com o filho recém-nascido e a redução da medicalização e intervenções tecnológicas foram alguns dos principais pontos para os quais essas recomendações focaram. Este documento, também conhecido como Declaração de Fortaleza, tornou-se uma fonte legítima no campo da assistência ao parto e grupos ativistas em diferentes partes do mundo (ARAUJO MMT e SILVA MJP, 2017).

No entanto, algumas destas recomendações nem sempre são bem recebidas pelos profissionais em todas as partes do mundo, pode impactar negativamente a assistência obstétrica fornecida e levar a graves violações de direitos reprodutivos. Argumenta-se que a abordagem de Saúde Sexual e Direitos Sociais Reprodutivos e o consenso da OMS influenciaram mudanças nas abordagens institucionais para assistência à saúde reprodutiva e ao parto - algumas que se refletem em textos legais de violência obstétrica adotados pela Venezuela, Argentina Brasil (CAMPOS VS, et al., 2020).

Como não há literatura, especificamente, resumindo essas abordagens legais e institucionais para parto, a última seção deste capítulo será a primeira tentativa de apresentar uma revisão legal da violência obstétrica, concentrando-se em proporcionar uma melhor compreensão dos termos relacionados com o parto, questões de gênero, negligência médica e a ética da assistência à saúde (BOURDIEU P, 1971).

Defende-se que é importante rever este texto porque a recente introdução do conceito de "violência obstétrica" para denotar práticas puníveis sob as leis do Brasil indica uma mudança crucial na atenção governamental à assistência ao parto (BRASIL, 2010).

A abordagem legal tomada por esses países tem fornecido ativistas de parto globalmente com uma forma legítima de relatar situações indesejáveis vivenciadas por mulheres grávidas quando elas dão à luz hospitais. É uma ferramenta com muito potencial, como se ilustrara neste estudo (PASCHE DF, et al.,2010).

No Brasil, vários termos são usados para descrever o fenômeno: violência de gênero em nascimento e aborto; violência no parto; abuso obstétrico; violência institucional de gênero no parto e aborto; crueldade em parto; desumano / desumanizada assistência; violações dos direitos humanos de mulheres no parto; desrespeito e maus-tratos durante o parto. Essa definição permitiu a busca de estudos que abordem a emitir e contribuir para a formulação de estratégias para lidar com a violência obstétrica, como a promoção de legislação garantia dos direitos das mulheres (CUNHA AL, et al., 2019).

É necessário cuidar dos próprios profissionais da área da saúde, provendo melhores condições de trabalho para esses, com o intuito de proporcionar um melhor atendimento aos usuários da saúde, pois os profissionais precisam ser humanizados para verem os seus pacientes como humanos também (PROGIANTI JM, et al., 2019). 
As vezes a instabilidade emocional desenvolvida pela mulher está ligado ao fato de terem passados por experiência frustrante que as levam para tal estado emocional, relacionado ao estresse psicólogos que ocorrem durante o parto e podem desenvolver a depressão pós-parto, que resultou da intensa violência que sofreram (NASCIMENTO SL, et al., 2019).

\section{CONSIDERAÇÕES FINAIS}

É necessário o treinamento de profissionais da saúde para promover um atendimento humanizado e adequado para cuidados de saúde da mulher. Portanto, há necessidade de estudos que destacar a questão entre os profissionais de saúde, com ênfase nas boas práticas incluídas nele e conformidade e conformidade com leis, punições e formas de denúncia, para promover a reflexão sobre eles, com tendo em vista uma possível transformação na sua prática, apresentando formas não violentas e não impostas de estabelecer autoridade criando um vínculo baseado em respeito e segurança em relação às técnicas utilizado durante 0 período, seja no trabalho, parto ou no puerpério.

\section{REFERÊNCIAS}

1. ALTHABE F, et al. Abehavioral intervention to improve obstetrical care. The New England Journal of Medicine 2014; 512(43).

2. ARAÚJO MMT e. SILVA MJP. A comunicação com o paciente em cuidados paliativos: valorizando a alegria e o otimismo. Revista da Escola de Enfermagem da USP, 2017; 49(9).

3. BRASIL. Ministério da Saúde (BR). Parto, aborto e puerpério: assistência humanizada à mulher. 1ํed. Brasília (DF); 2010.

4. LANSKY S. Gestão de qualidade e da integralidade do cuidado em saúde para a mulher e a criança no SUS-BH: a experiência da comissão perinatal. Rev Tempus Actas Saúde Col, 2010; 4(4): 191-199.

5. PASCHE DF, et al. Humanização da atenção ao parto e nascimento no Brasil: pressuposto para uma nova ética na gestão e no cuidado. Revista Tempus Actas Saúde Coletiva, Brasília, 2010; 4(4): 105-117.

6. PAULETTI M, et al. Violência obstétrica: manifestações postadas em grupos virtuais no Facebook. Enfermeria (Montev.), 2020; 9(1): 3-20.

7. CAMPOS VS, et al. Práticas convencionais do parto e violência obstétrica sob a perspectiva de puérperas. Rev. baiana enferm, 2020; 34(1).

8. CURI PL, et al. A violência obstétrica praticada contra mulheres negras no SUS. Arq. bras. psicol. (Rio J. 2003), 2020; 72(1): 156-169.

9. MENEZES FR, et al. O olhar de residentes em Enfermagem Obstétrica para o contexto da violência obstétrica nas instituições. Interface (Botucatu, Online), 2020; 24(1).

10. SILVA TM, et al. Violência obstétrica: a abordagem da temática na formação de enfermeiros obstétricos. Acta Paul. Enferm. (Online), 2020; 33(1).

11. SOUZA ACAT, et al. Violência obstétrica: uma revisão integrative. Rev. enferm. UERJ, 2019; 27(1).

12. MARQUES GM e NASCIMENTO DZ. Alternativas que contribuem para a redução da violência obstétrica. Ciênc. Saúde Colet, 2019; 24(12): 4743-4744.

13. NASCIMENTO SL, et al. Conhecimentos e experiências de violência obstétrica em mulheres que vivenciaram a experiência do parto. Enferm. actual Costa Rica (Online), 2019; (37): 66-79.

14. OLIVEIRA LLF, et al. Violência obstétrica em serviços de saúde: constatação de atitudes caracterizadas pela desumanização do cuidado. Rev. enferm. UERJ, 2019; 27(1).

15. OLIVEIRA MSS, et al. Vivências de violência obstétrica experimentadas por parturientes. ABCS health sci, 2019; 44(2): 114-119.

16. MARQUES SB. Violência obstétrica no Brasil: um conceito em construção para a garantia do direito integral à saúde das mulheres. Cad. Ibero Am. Direito Sanit., 2020; 9(1).

17. TEIXEIRA PC, et al. Percepção das parturientes sobre violência obstétrica: a dor que querem calar. Revista Nursing, 2020; 23(261): 3607-3615.

18. CUNHA AL, et al. Produção de conhecimento sobre violência obstétrica: o lado invisível do parto. Revista Nursing, 2019; 23(260): 3529-3532.

19. CASTRO ATB e ROCHA SP. Violência obstétrica e os cuidados de enfermagem: reflexões a partir da literatura. Revista Oficial do Conselho Federal de Enfermagem, 2020; 11(1): 176-181.

20. PROGIANTI JM, et al. Vivências de sofrimento e prazer das acadêmicas de enfermagem nas maternidades. Rev. enferm. UERJ, 2019; 27(1). 